\title{
Mitglieder der TNM-Komitees der UICC
}

1950 bestellte die UICC ein Committee on Tumour Nomenclature and Statistics. 1954 wurde dieses Komitee in Committee on Clinical Stage Classification and Applied Statistics umbenannt; seit 1966 nennt es sich Committee on TNM Classification. Die in diesen Komitees tätigen Mitglieder sind nachfolgend aufgeführt:

Anderson, W.A. D.

Baclesse, F.

Badellino, F.

Barajas-Vallejo, E.

Blinov, N.

Bucalossi, P.

Burn, I.

Bush, R.S.

Carr, D.T.

Copeland, M.M.

Costachel, O.

Denoix, $\mathrm{P}$.

Fischer, A. W.

Gentil, F.

Ginsberg, R.

Hamperl, $\mathrm{H}$.

Harmer, M. H.

Hayat, M.

Hermanek, P.

Hultberg, S.

Hutter, R. V.P.

Ichikawa, $\mathrm{H}$.

Imai, T.
USA

Frankreich

Italien

Mexiko

UdSSR

Italien

Vereinigtes Königreich

Kanada

USA

USA

Rumänien

Frankreich

Bundesrepublik Deutschland

Brasilien

Kanada

Bundesrepublik Deutschland

Vereinigtes Königreich

Frankreich

Bundesrepublik Deutschland

Schweden

USA

Japan

Japan 
Ishikawa, S.

Junqueira, A.C.C.

Kasdorf, $\mathbf{H}$.

Kottmeier, H. L.

Koszarowski, T.

Levene, A.

Lima-Basto, E.

Logan, W.P. D.

McWhirter, $R$.

Perazzo, D. L.

Perez-Modrego, S.

Perry, I. H.

Rakov, A.I.

Roxo-Nobre, M.O.

Sellers, A. H.

Sobin, L. H.

Spiessl, B.

Van der Werf-Messing, B.

Wagner, R. I.

Watson, T.A.
Japan

Brasilien

Uruguay

Schweden

Polen

Vereinigtes Königreich

Portugal

Vereinigtes Königreich

Vereinigtes Königreich

Argentinien

Spanien

USA

UdSSR

Brasilien

Kanada

USA

Schweiz

Niederlande

UdSSR

Kanada 